\title{
Endoscopic urethrotomy versus open urethroplasty for men with bulbar urethral stricture: the OPEN randomised trial cost-effectiveness analysis
}

Jing Shen ${ }^{1}$, Luke Vale ${ }^{1 *}$, Beatriz Goulao ${ }^{2}$, Paul Whybrow ${ }^{3}$, Stephen Payne ${ }^{4}$ and Nick Watkin ${ }^{5}$ on behalf of OPEN trial investigators

\begin{abstract}
Background: Bulbar urethral stricture is a common cause for urinary symptoms in men and its two main treatment options both have drawbacks with little evidence on their relative cost-effectiveness. Current guidelines on the management of recurrent bulbar urethral stricture have been predominantly based on expert opinion and panel consensus.

Objective: To assess the relative cost-effectiveness of open urethroplasty and endoscopic urethrotomy as treatment for recurrent urethral stricture in men.

Methods: Set in the UK National Health Service with recruitment from 38 hospital sites, a randomised controlled trial of open urethroplasty and endoscopic urethrotomy with 6-monthly follow-up over 24 months was conducted. Two hundred and twenty-two men requiring operative treatment for recurrence of bulbar urethral stricture and having had at least one previous intervention for stricture were recruited. Effectiveness was measured by quality- adjusted life years (QALYS) derived from EQ-5D 5L. Cost-effectiveness was measured by the incremental cost per QALY gained over 24 months using a within trial analysis and a Markov model with a 10-year time horizon.

Results: In the within trial, urethroplasty cost on average more than urethrotomy (cost difference: $£ 2148$ [95\% Cl $689,3606]$ ) and resulted in a similar number of QALYs on average (QALY difference: -0.01 [95\% Cl - 0.17, 0.14)] over 24 months. The Markov model produced similar results. Sensitivity analyses using multiple imputation, suggested that the results were robust, despite observed missing data.
\end{abstract}

Conclusions: Based on current practice and evidence, urethrotomy is a cost-effective treatment compared with urethroplasty.

Keypoints: Urethrotomy and urethroplasty both led to symptom improvement for men with bulbar urethral stricture-a common cause for urinary symptoms in men; Urethroplasty appeared unlikely to offer good value for money compared to urethrotomy based on current evidence.

Trial registration: ISRCTN: 98009168 (date: 29 November 2012) and it is also in the UK NIHR Portfolio (reference 13507).

\footnotetext{
*Correspondence: luke.vale@newcastle.ac.uk

1 Population Health Sciences Institute, Newcastle University, Newcastle

upon Tyne, UK

Full list of author information is available at the end of the article
}

(C) The Author(s) 2021. Open Access This article is licensed under a Creative Commons Attribution 4.0 International License, which permits use, sharing, adaptation, distribution and reproduction in any medium or format, as long as you give appropriate credit to the original author(s) and the source, provide a link to the Creative Commons licence, and indicate if changes were made. The images or other third party material in this article are included in the article's Creative Commons licence, unless indicated otherwise in a credit line to the material. If material is not included in the article's Creative Commons licence and your intended use is not permitted by statutory regulation or exceeds the permitted use, you will need to obtain permission directly from the copyright holder. To view a copy of this licence, visit http://creativecommons.org/licenses/by/4.0/. The Creative Commons Public Domain Dedication waiver (http://creativeco mmons.org/publicdomain/zero/1.0/) applies to the data made available in this article, unless otherwise stated in a credit line to the data. 
Trial protocol: The latest version (1.8) of the full protocol is available at: www.journalslibrary.nihr.ac.uk/programmes/ hta/105723/\#/ and a published version is also available: Stephenson R, Carnell S, Johnson N, Brown R, Wilkinson J, Mundy A, et al. Open urethroplasty versus endoscopic urethrotomy-clarifying the management of men with recurrent urethral stricture (the OPEN trial): study protocol for a randomised controlled trial. Trials 2015;16:600. https://doi. org/10.1186/s13063-015-1120-4.

Trial main clinical results publication: Goulao B, Carnell S, Shen J, MacLennan G, Norrie J, Cook J, et al. Surgical Treatment for Recurrent Bulbar Urethral Stricture: A Randomised Open-label Superiority Trial of Open Urethroplasty Versus Endoscopic Urethrotomy (the OPEN Trial), European Urology, Volume 78, Issue 4, 2020, Pages 572-580.

Keywords: Cost-effectiveness, Economic model, Randomised controlled trial, Urethral stricture, Urethroplasty, Urethrotomy

\section{Introduction}

Bulbar urethral stricture is a common cause for urinary symptoms (typically difficulty in passing urine) in men. Initial treatment is usually by endoscopic urethrotomy, a procedure that produces widening of the narrowed urethral segment by incising the stricture internally under vision. In about $50 \%$ of the cases the stricture will recur requiring re-treatment [1]. This can be via a repeat endoscopic urethrotomy, graduated dilatation or formally repaired by urethroplasty with either excision of the stricture and anastomosis, or augmentation using a graft such as buccal mucosa.

Urethrotomy is most commonly performed for recurrent bulbar stricture because it is minimally invasive, does not require specialist surgical expertise, and has a short period of urethral catheterisation and recovery. However, further recurrence is likely [2]. Open urethroplasty is more invasive, requires specialist expertise, a longer period of catheterisation and a more protracted return to normal activities. Nevertheless, urethroplasty may offer the prospect of long-term cure without the need for further interventions [3, 4]. When men are choosing between these two options, they have to make a trade-off between the invasiveness and effectiveness of each operation [1,5-8]. Current decision-making is therefore determined by the availability of local expertise, clinician guidance, patient co-morbidity, and patient preferences.

The guidelines from the American Urological Association (AUA) and Société Internationale d'Urologie (SIU) recommend treatment with endoscopic techniques for first time bulbar strictures [1]. For recurrent strictures the guideline recommendations differ, with the AUA recommending urethroplasty and the SIU recommending endoscopic approaches or urethroplasty if symptomatic recurrence is after more than 3 months [1]. The reviews underpinning published guidelines, sponsored jointly by the SIU, the International Consultation on Urologic Disease [1,5] and by the AUA [7], included non-randomised studies, predominantly of retrospective cohort design. Consequently the formulation of the guideline recommendation subsequent to these reviews was predominantly based on expert opinion and panel consensus rather than any robust evidence of the relative effectiveness or cost-effectiveness of these two treatment options.

To address this evidence gap, a randomised controlled trial (RCT) was conducted to compare the clinical and cost-effectiveness of urethroplasty versus endoscopic urethrotomy for the alleviation of urinary symptoms in men with recurrent bulbar stricture over 24 months (the OPEN (open urethroplasty versus endoscopic urethrotomy) Trial [6]). Clinical results of the OPEN trial are presented elsewhere [9]. This paper presents the costeffectiveness analysis results.

\section{Methods}

OPEN was a pragmatic patient-randomised two-arm superiority trial, which recruited across 38 National Health Service (NHS) secondary care providers in the UK. The algorithm allocated participants to each intervention in a 1:1 ratio with recruitment site and time since last procedure ( $<12$ months or $\geq 12$ months) as minimisation covariates. Trial procedures and statistical analysis are described elsewhere $[6,9]$.

The OPEN trial's economic analysis comprised a within trial cost-effectiveness analysis and Markov model with a 10-year time horizon. The within trial analysis estimated the costs from a societal perspective, quality adjusted life years (QALYs) gained and incremental cost per QALY of open urethroplasty compared to endoscopic urethrotomy over a 24-month period. As urethroplasty was a priori expected to be both more effective and more costly than endoscopic urethrotomy, with its benefits persisting beyond 24 months, Markov modelling [10] was conducted to examine the relative efficiency with a 10-year time horizon. Costs were reported as pound Sterling for the price year 2017. Where costs came from different years they were converted to 2017 values using the Consumer Price Index [11], as this was the last full year when unit costs were available before the analysis 
was conducted. All costs and QALYs were appropriately discounted using the recommended discount rate $3.5 \%$ per annum) [12]. Sensitivity analysis [13] was conducted to address uncertainty in study parameters and multiple imputation [14] was adopted to deal with missing data.

\section{Resource use and cost}

Micro costing methods [15] were used to estimate the cost of the two interventions on a per patient basis. The cost of the interventions included the staff involved, the use of reusable and disposable equipment and use of the theatre suite. Data that varied by participant were collected on the trial's Case Report Form, whilst other data came from communications with clinicians at the main trial site (Freeman hospital, Newcastle upon Tyne, UK). The unit costs of these resources came from standard sources [16-18].

When trial participants had a re-intervention during the trial's follow-up period, the same process of micro costing used to cost the index procedure was invoked. Other use of primary and secondary NHS services, medications and participants' out-of-pocket expenses relating to the condition came from the Case Report Form and a bespoke participant cost questionnaire completed at six-monthly intervals. Unit costs came from standard sources and participant cost questionnaire.

\section{Quality-adjusted life years}

QALYs were based on responses to the EQ-5D-5L collected at baseline, immediately prior to surgery, 1 week after catheter removal, 3, 6, 9, 12, 24 months following surgery, 18 and 24 months after randomisation and at the end of study. The responses to the EQ-5D-5L questionnaire were scored using UK population tariffs [19] to produce a health state utility score for each participant in each of the treatment groups using the area under the curve (AUC) method [20].

Given the large number of time points for EQ-5D-5L data and to align with the primary effectiveness analysis [9], it was decided that to be included in the AUC analysis as a complete case, the participant must have at least three EQ-5D-5L observations with one at the start of the assessment period, one at the mid-range and one at the end. The specific requirements depended on whether the participants had an initial intervention and the type of analysis conducted (details in the note under Table 2).

For all calculations of QALYs, the first observation used was set at time point zero and the actual date of completion for each individual EQ-5D-5L questionnaire was used to calculate the number of days from the first observation. In the sensitivity analyses, QALYs were rescaled to the nominal data collection points, i.e. 730 days, to account for difference in waiting times between the two interventions (note that cost data did not require rescaling in the same way as the recall period, because it was pre-defined within the data collection tools). Additionally, multiple imputation for EQ-5D-5L at all missing time points was conducted to calculate QALYs for all participants.

\section{Within trial cost-utility analysis}

The cost-utility analysis used an intention-to-treat principle. The incremental cost-effectiveness ratio (ICER) was calculated by dividing the difference in mean costs by the difference in mean QALYs for each group. Results were presented as point estimates of the mean incremental costs, QALYs and cost per QALY, estimated using seemingly unrelated regression [21], controlling for dichotomised time since last procedure (less than 12 months, 12 months or more), allocated treatment arm and baseline utility. Multiple imputation was performed to complete any missing data and used in the sensitivity analysis. Stochastic uncertainty in parameter estimates was addressed through the application of bootstrapping and the estimation of cost-effectiveness acceptability curves.

\section{Markov model}

The Markov model consisted of three health statessymptom-free, symptomatic and deceased-in care pathways describing the process of care and disease incidence and progression. The base case analysis used parameters that were estimated based on information given by study participants who were allocated and received the allocated treatment. Both probabilistic and deterministic sensitivity analyses were conducted to address uncertainty. Deterministic sensitivity analyses included basing model parameters on those who received the same treatment procedure regardless of their allocated intervention group (due to cross-over in the trial) and varying the probabilities of follow-up intervention conditional on the previous intervention. The parameters used to populate the Markov model are reported in supplementary material (Additional file 1: Table S1). Model structure is shown in supplementary material (Additional file 1: Figure S3).

\section{Results}

A total of 222 men were randomised, two of whom were excluded from analysis post-randomisation because further assessment prior to intervention found them to be ineligible. 108 were in the urethroplasty group and 112 in the urethrotomy group.

\section{Costs}

Total costs combining NHS resource use costs (intervention, re-intervention and health service use 
during follow-up) and patients' out-of-pocket costs are presented in Table 1 . The cost of urethroplasty was statistically significantly higher over 24 months post randomisation than that of urethrotomy, with the cost difference ranging between $£ 1333$ and $£ 2123$ depending on whether follow-up care and patient costs were included for the base care and sensitivity analyses.

\section{Quality-adjusted life years}

Estimates of QALYs for the base case analysis and all the sensitivity analyses are presented in Table 2. Urethrotomy in general appears to generate higher QALYs than urethroplasty. However, the difference was not statistically significant apart from evidence of a slight difference for the rescaled QALYs at 24 months after surgery $(p<0.1)$ and the rescaled QALYs with imputation $(p<0.1)$.

\section{Within trial cost-utility analysis}

In the base case, urethroplasty costed more than urethrotomy while generating a lower QALY, therefore, was dominated by urethrotomy (Table 3 ). The base case results appeared robust as they were similar in the sensitivity analyses (Table 3). The cost-effectiveness acceptability curve (Fig. 1) and incremental cost and QALY plots (Additional file 1: Figure S1) are presented for the base case.

\section{Markov model}

In the base case analysis, urethroplasty is unlikely to be considered cost-effective under current society's willingness to pay threshold for a QALY and this is supported by the sensitivity analyses results (Table 4 and Figs. 2 and Additional file 1: Figure S2). This is mainly due to the higher cost of urethroplasty compared to urethrotomy, whilst both of the treatment options produce similar QALY gains. This is despite those receiving urethroplasty

Table 1 Total cost (£) for each trial group

\begin{tabular}{|c|c|c|c|c|}
\hline \multirow[t]{2}{*}{ Total cost } & \multicolumn{2}{|c|}{ Urethroplasty } & \multicolumn{2}{|l|}{ Urethrotomy } \\
\hline & Mean (SD) $£$ & $\mathbf{N}$ & Mean (SD) $f$ & $\mathbf{N}$ \\
\hline \multicolumn{5}{|l|}{ Base case } \\
\hline Total intervention and re-intervention cost & $4332(3151)$ & 89 & $2209(2368)$ & 91 \\
\hline Total NHS cost (intervention and re-intervention with follow-up) & $4455(3191)$ & 89 & $2657(3476)$ & 91 \\
\hline Total societal cost (NHS and patient costs) & $4480(3218)$ & 89 & $2730(3713)$ & 91 \\
\hline \multicolumn{5}{|l|}{ Sensitivity analysis with data imputation } \\
\hline Total intervention and re-intervention cost & $4559(3061)$ & 108 & $2911(2713)$ & 112 \\
\hline Total NHS cost (intervention and re-intervention with follow-up) & $4674(3135)$ & 108 & $3310(3552)$ & 112 \\
\hline Total societal cost (NHS and patient costs) & $4704(3155)$ & 108 & 3371 (3755) & 112 \\
\hline
\end{tabular}

Table 2 Utility values at each time point and QALYs over the trial follow-up

\begin{tabular}{|c|c|c|c|c|}
\hline \multirow[t]{2}{*}{ EQ-5D 5L } & \multicolumn{2}{|c|}{ Urethroplasty } & \multicolumn{2}{|c|}{ Urethrotomy } \\
\hline & Mean (SD) & $\mathbf{N}$ & Mean (SD) & $\mathbf{N}$ \\
\hline QALYs at 24 months after randomisation & $1.75(0.40)$ & 55 & $1.76(0.35)$ & 54 \\
\hline QALYs at 24 months after randomisation (rescaled to 730 days) & $1.66(0.34)$ & 55 & $1.70(0.34)$ & 54 \\
\hline QALY at 24 months after surgery & $1.73(0.54)$ & 44 & $1.77(0.34)$ & 56 \\
\hline QALY at 24 months after surgery (rescaled to 730 days) & $1.42(0.40)$ & 44 & $1.58(0.30)$ & 56 \\
\hline QALY at 24 months after randomisation with imputation & $1.73(0.32)$ & 108 & $1.76(0.28)$ & 112 \\
\hline QALY at 24 months after randomisation with imputation (rescaled to 730 days) & $1.67(0.29)$ & 108 & $1.72(0.27)$ & 112 \\
\hline QALY at 24 months after surgery with imputation & $1.75(0.37)$ & 108 & $1.76(0.29)$ & 112 \\
\hline QALY at 24 months after surgery with imputation (rescaled to 730 days) & $1.67(0.30)$ & 108 & $1.72(0.26)$ & 112 \\
\hline
\end{tabular}

QALY calculations For those participants who did not receive an initial intervention, to be included in the AUC analysis without imputation, they must have complete EQ-5D-5L data on all of the three time points: baseline, 18 months and 24 months after randomisation. For those participants who received an initial intervention, the base case analysis examined QALY over the period from baseline to 24 months after randomisation, therefore, the base case AUC analysis required complete EQ-5D-5L data at baseline and 24 months after randomisation, and at one of the data collection points of 3,6, 9, 12 following surgery and 18 months following randomisation. Given the differences in the time lengths between randomisation and undergoing an intervention between urethroplasty and urethrotomy, sensitivity analyses also examined QALY over the period from the time prior to surgery to 24 month post-surgery, in which case the AUC analysis required complete EQ-5D-5L data at prior to surgery and 24 months after surgery, and at one of the data collection points of $3,6,9,12$ following surgery and 18,24 months following randomisation 


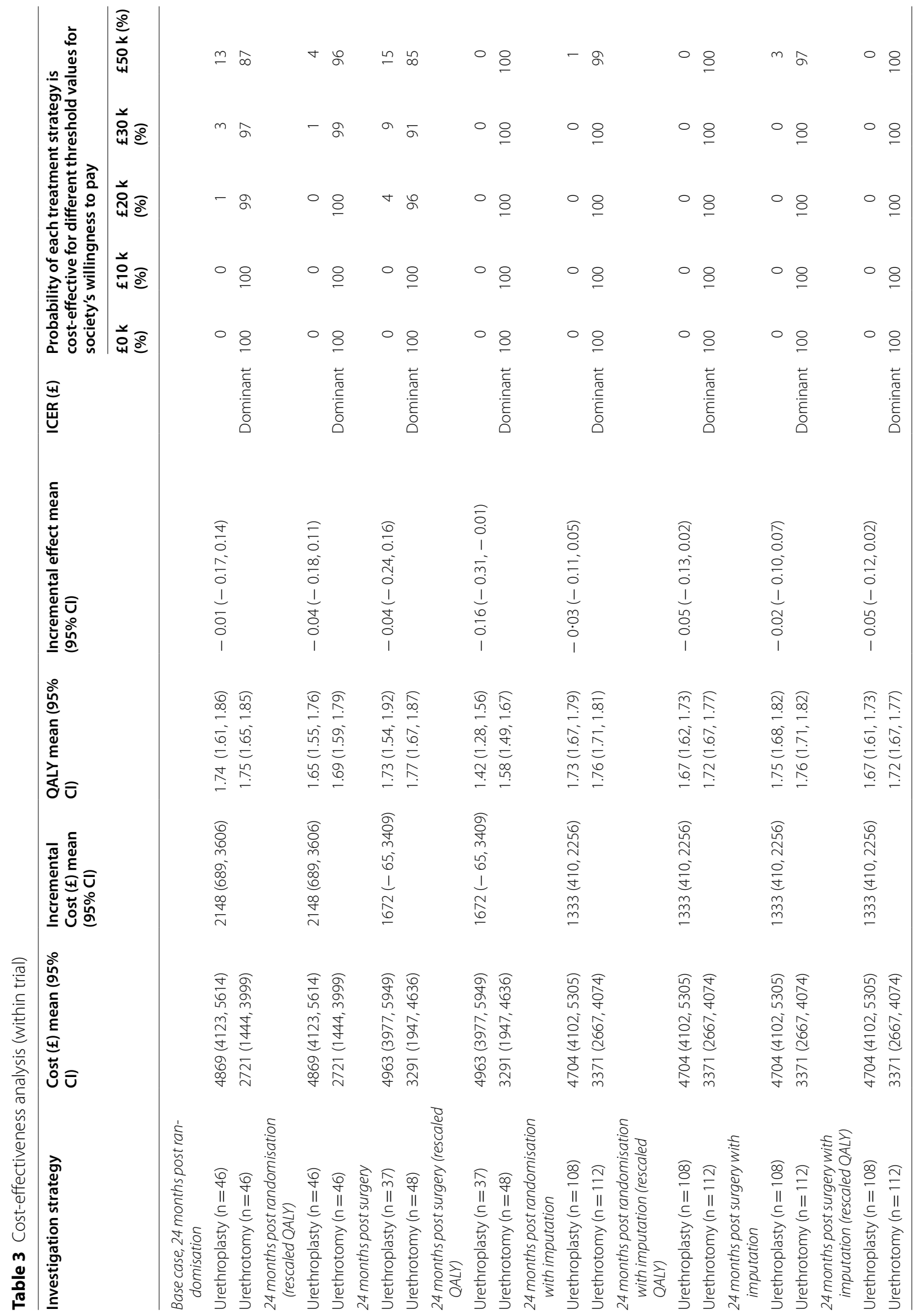




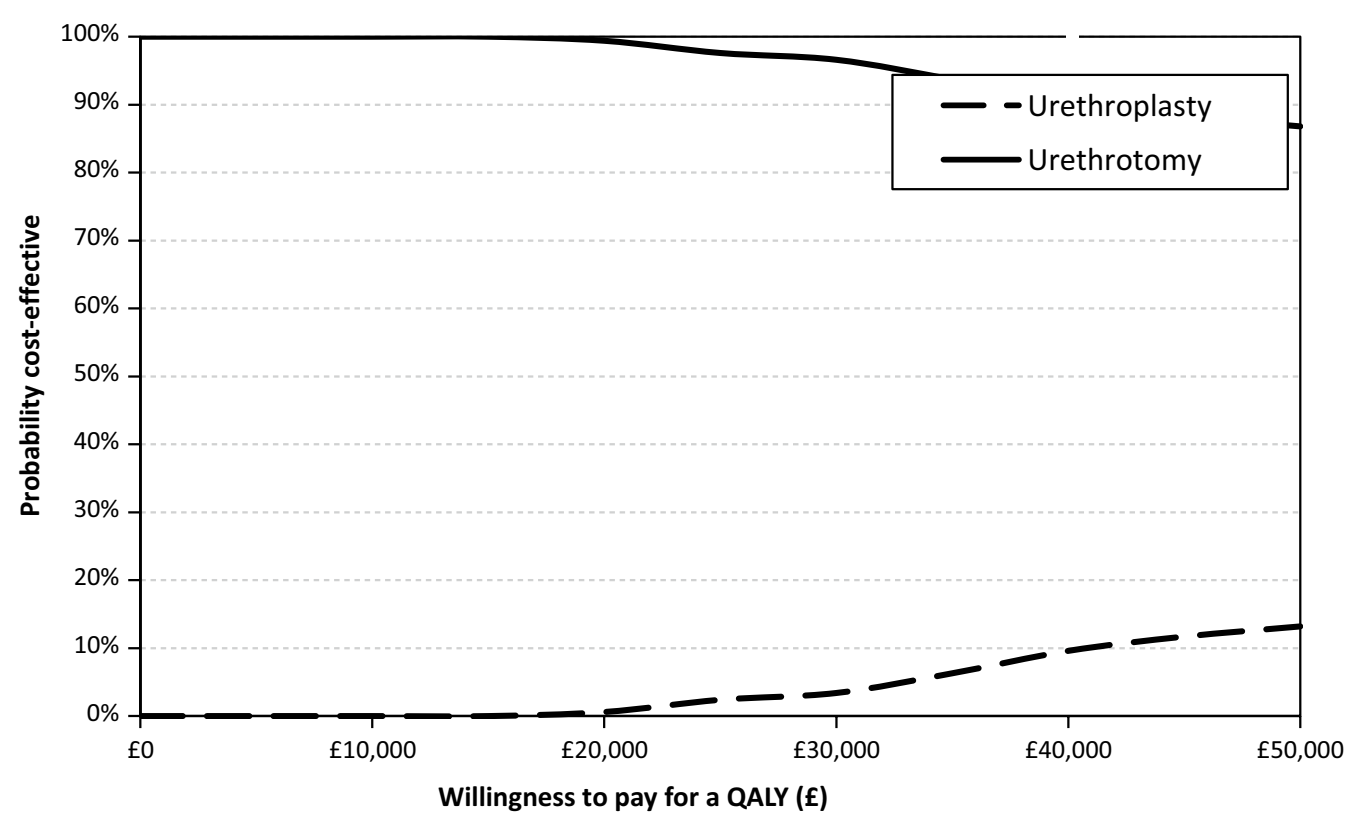

Fig. 1 Cost-effectiveness acceptability curve (Base case)

Table 4 Markov model result over 10 years

\begin{tabular}{|c|c|c|c|c|c|c|c|c|c|}
\hline \multirow[t]{2}{*}{ Analyses } & \multirow[t]{2}{*}{$\begin{array}{l}\text { Treatment } \\
\text { strategy }\end{array}$} & \multirow[t]{2}{*}{$\begin{array}{l}\text { Cost } \\
\text { (£) }\end{array}$} & \multirow[t]{2}{*}{ QALY } & \multirow[t]{2}{*}{$\begin{array}{l}\text { ICER } \\
(f)\end{array}$} & \multicolumn{5}{|c|}{$\begin{array}{l}\text { Probability of each treatment strategy is } \\
\text { cost-effective for different threshold values for } \\
\text { society's willingness to pay for a QALY }\end{array}$} \\
\hline & & & & & $\begin{array}{l}£ 0 \mathrm{k} \\
(\%)\end{array}$ & $\begin{array}{l}£ 10 k \\
(\%)\end{array}$ & $\begin{array}{l}£ 20 \mathrm{k} \\
(\%)\end{array}$ & $\begin{array}{l}£ 30 \mathrm{k} \\
(\%)\end{array}$ & $\begin{array}{l}£ 50 \mathrm{k} \\
(\%)\end{array}$ \\
\hline \multirow[t]{2}{*}{ Base case } & Urethroplasty & 8026 & 7.61 & 301,073 & 0 & 0 & 0 & 0 & 2 \\
\hline & Urethrotomy & 6553 & 7.60 & & 100 & 100 & 100 & 100 & 98 \\
\hline \multirow[t]{2}{*}{ Parameters based on treatment received } & Urethroplasty & 7987 & 7.61 & 307,328 & 0 & 0 & 0 & 0 & 1 \\
\hline & Urethrotomy & 6490 & 7.60 & & 100 & 100 & 100 & 100 & 99 \\
\hline \multirow[t]{2}{*}{ Always receive the same treatment at recurrence } & Urethroplasty & 9026 & 7.61 & 476,162 & 0 & 0 & 0 & 0 & 0 \\
\hline & Urethrotomy & 4059 & 7.60 & & 100 & 100 & 100 & 100 & 100 \\
\hline \multirow[t]{2}{*}{ Always receive the other treatment at recurrence } & Urethroplasty & 8076 & 7.61 & 263,383 & 0 & 0 & 1 & 2 & 4 \\
\hline & Urethrotomy & 7054 & 7.60 & & 100 & 100 & 99 & 98 & 96 \\
\hline
\end{tabular}

having a lower chance of recurrence compared to those receiving urethrotomy.

\section{Discussion}

Relative efficiency within the trial follow-up and over a 10-year time horizon was found to favour urethrotomy compared with urethroplasty. Effectiveness as measured by QALYs appeared to be broadly equivalent between the two trial arms of the OPEN trial, and this was in line with the primary clinical outcome finding showing no statistically significant difference in the primary patient reported outcome between trial arms [9]. Costs were higher in the urethroplasty arm than the urethrotomy arm. Therefore, urethroplasty had a higher cost whilst producing similar QALY gain, making it unlikely to be cost-effective, despite having a lower chance of recurrence compared to urethrotomy.

The relatively high cost of urethroplasty is the main reason that it was not as cost-effective. It may be possible to reduce the cost of urethroplasty by using daycare surgery [22], a shorter period of catheterisation [23] or by rationalising follow-up to those most likely to need it and discharging symptom stable patients at an early stage [24]. Length of stay following urethroplasty within the trial was 1.34 (SD 0.95) days vs 0.52 (SD 1) for urethrotomy [25]. Therefore, scope to substantially reduce length of stay is limited and even if length of 


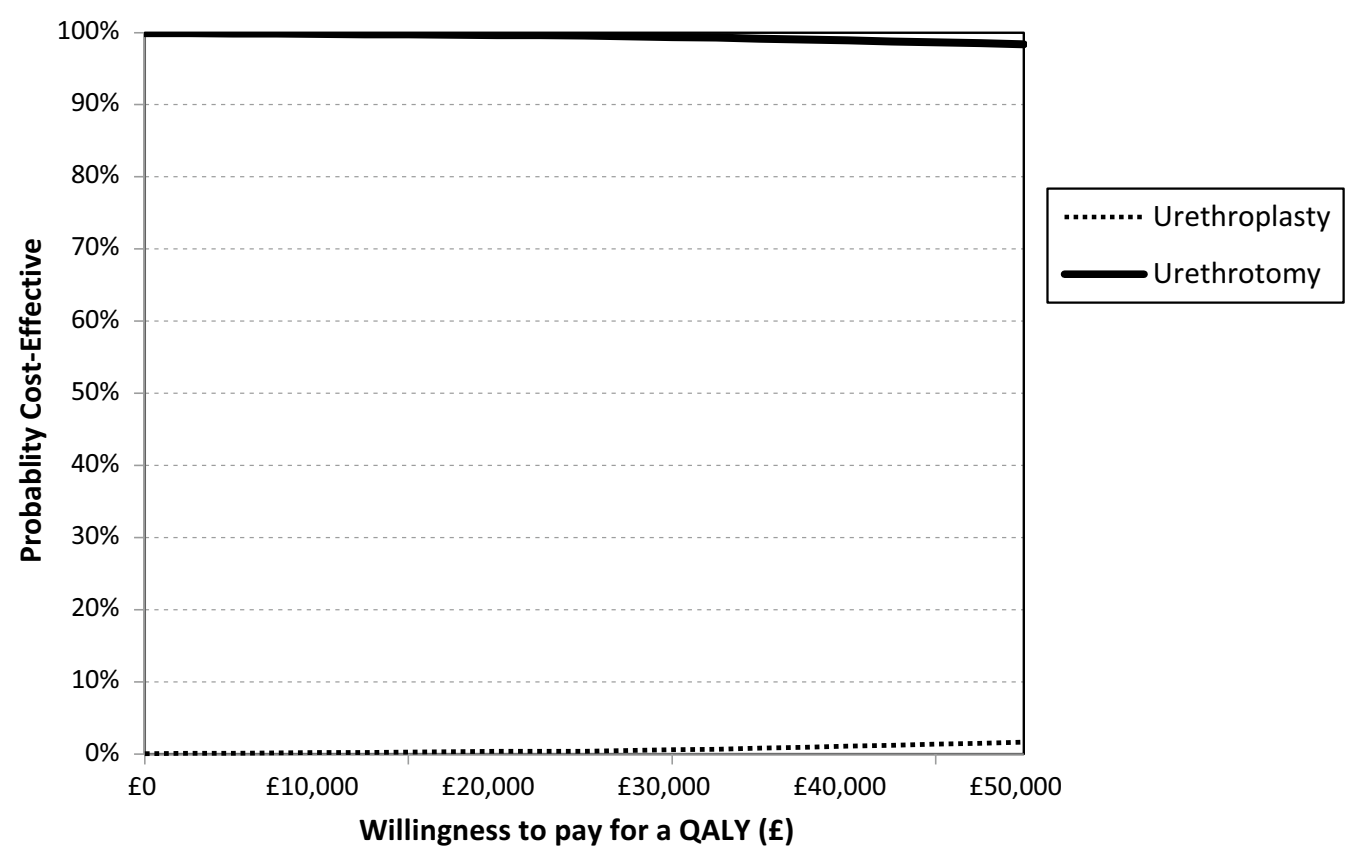

Fig. 2 Cost-effectiveness acceptability curve (Markov model base case)

stay was reduced to zero this would be insufficient to reduce the cost of urethroplasty to close to the cost of urethrotomy.

However, the higher cost of urethroplasty is driven by the higher theatre procedure and it is unlikely that theatre time could be reduced significantly to alleviate the increased costs of urethroplasty. Hospitalisation accounts for around $15 \%$ of the total intervention cost. Strategies to reduce length of stay could be explored as a way of lowering the cost of urethroplasty. In Europe, practice varies widely with length of stay ranging from 2 to 7 days in high volume centres exaggerating the difference between the two procedures costs. The mean length of stay in the UK trial was 2 days. It is unlikely that further substantial reductions in length of stay would be possible, although pre-operative counselling, improved perioperative analgesia and discharge education could help reduce this to 1.5 days.

Although the study showed no difference in effectiveness as measured by QALYs, it is worth noting that the loss of quality of life during recovery from subsequent procedures was not taken into consideration. Given the likelihood of recurrence requiring repeated treatments, this decrement in quality of life could make a difference in total QALYs between the two treatments. Patients undergoing urethroplasty are also less likely to have recurrence in the long-term based on the observation during the trial's follow-up period and consequently suffer less loss in quality of life and hence may accrue more
QALYs over time. However, there was no obvious signal of reductions in quality of life caused by differences in recurrent rates [25]. However, this requires further study. Additionally, EQ-5D as a generic quality of life measure may not be sensitive enough to capture changes in health-related quality of life among patients with bulbar urethral stricture, as the impact on quality of life from the condition may fall mostly around the time of recurrence or only present in one or two dimensions (e.g. pain, anxiety) of EQ-5D. Future research should focus on how best to capture quality of life loss during recurrence and exploring alternative measures for this type of patients using preference elicitation methods [26, 27].

When examining the cost-effectiveness over the longterm, a key uncertainty in the modelling was the choice of re-intervention. The study data showed a large proportion of patients switched to a treatment different from their previous treatment when they had a re-intervention. There is no consensus on treatment choices for reinterventions, and such choices are often influenced by many non-clinical factors such as patient choice, waiting time, and travel time [8]. However, varying the probabilities of treatment options conditional on the previous intervention in the sensitivity analyses conducted for the modelling showed that urethroplasty remained less likely to be cost-effective.

Due to the trial's follow-up period of two years, we could potentially be underestimating the costs of the urethrotomy arm, however, questions arise on how 
many recurrences there need to be to offset the more expensive urethroplasty and over what length of time. On the other hand, there was little evidence suggesting patients' quality of life differed significantly between the two arms, at least within the trial's follow-up period. We do not claim that cost is the only concern in the choice of treatment, but in the absence of clear evidence on the additional benefits one treatment can bring, costs may be an important factor to consider in a publicly funded health system. These issues were also extrapolated over 10 years using a Markov model, which met or exceeded internationally accepted guidelines for best practice for the conduct of such work. This analysis showed that the initial higher costs of urethroplasty were not likely to be offset by increased reintervention rates but that urethroplasty was on average more effective.

The key strength of the present study is the use of an RCT designed to detect the clinically meaningful difference in voiding symptom score. The RCT design was based on current best practice for pragmatic surgical trials and it sought to provide direct and most up to date information on costs and utilities. However, obtaining data directly from a large clinical study also has its downside. The principle limitation of the RCT was the missing data caused by trial participants being lost to follow-up; a common situation in a complex study involving several years of follow-up. We remedied this by using multiple imputation for the missing data and comparing complete case analysis with analyses using imputation. These sensitivity analyses demonstrated that the study results were robust.

Although the economic evaluation was conducted in the UK NHS, the results are generalisable to other countries with similarly public funded healthcare systems. As we have seen that there is little difference in QALYs between the two treatment options, regardless of unit costs of resources used, urethroplasty is generally more costly than urethrotomy due to the longer operating and recovery time. Therefore, the conclusions drawn for QALYs and costs would stay unchanged in other settings.

\section{Conclusions}

This paper has presented a comprehensive economic analysis of the relative efficiency of urethroplasty and urethrotomy within an RCT over 24 months and over a 10 -year time horizon. Based on current practice and evidence, urethroplasty is unlikely to be cost-effective due to its higher cost. Future research should examine how the benefits of reduced recurrence by urethroplasty can be captured while finding ways to reduce its costs.

\section{Abbreviations}

OPEN: Open urethroplasty versus endoscopic urethrotomy; NHS: National Health Service; QALY: Quality adjusted life years; AUC: Area under the curve; ICER: Incremental cost-effectiveness ratio; RCT: Randomised controlled trial.

\section{Supplementary Information}

The online version contains supplementary material available at https://doi. org/10.1186/s12894-021-00836-1.

Additional file 1. Additional material on the methods and results of the economic evaluation.

\section{Acknowledgements}

Contributors: Jing Shen, supervised by Luke Vale, led the health economic evaluation and analysis. Robert Pickard led the study, Beatriz Goulao supervised by Graeme MacLennan performed the statistical analysis, Sonya Carnell and Rebecca Forbes supported by Stephanie Currer and supervised by Jennifer Wilkinson managed the trial. John Norrie contributed to the funding application and statistical analysis plan. He also provided support to the statistical analysis team. Matt Breckons, supervised by Jing Shen, conducted a time trade off experiment reported separately. Paul Whybrow, supervised by Tim Rapley, carried out the qualitative research reported separately. Mark Forrest managed and maintained the trial database. Elaine McColl contributed to the funding application and protocol. Daniela Andrich, Anthony Mundy, James N'Dow and Stephen Payne provided clinician support to the funding application, acted as PIs at key sites. Stewart Barclay contributed a patient view to the trial, including the funding application, the protocol, and all aspects of the analysis. Jonathan Cook was involved in the design of the study and Nick Watkin co-wrote the funding application, contributed clinical insight to the trial management group. We thank the patients and health-care professionals for their participation in the study; Stewart Barclay, the patient and service user representative in the OPEN Trial Management Group; the Trial Steering committee members: Roger Kockelburg (chairperson), John Matthews, Alan McNeil, Howard Kynaston and Neil Campling; Data Monitoring Committee members: Gordon Murray (chairperson), Richard Martin and Thomas Pinkney. We also thank the following people who worked on the trial: Matthew Jackson, Research Fellow; Gladys McPherson, Data Manager; Lee Munro, Trial Manager; Rachel Stephenson, Trial Manager; Sue Tremble, Trial Manager; Robbie Brown, Trial Manager; Mark Deverill, Health Economist; Amy Collins, Project Secretary; Lavinia Miceli, Project Secretary; and Ann Payne, Project Secretary. Members of the Open Trial Group responsible for recruitment and who acted as principle investigators for their centres were: Trevor Dorkin, Freeman Hospital, Newcastle; Nick Watkin, St George's Hospital, London; Anthony Mundy, University College London Hospitals NHS Foundation Trust, London; Paul Anderson, Russells Hall Hospital, Dudley; Suzie Venn, Queen Alexandra Hospital, Portsmouth; Ian Eardley, St James's University Hospital, Leeds; Mr David Dickerson, Weston General Hospital, Weston-super-Mare; Nikesh Thiruchelvam, Addenbrooke's Hospital, Cambridge; Richard Inman and Chris Chapple, Royal Hallamshire Hospital, Sheffield; Andrew Baird, Aintree University Hospital NHS Foundation Trust, Liverpool; Andrew Sinclair, Stepping Hill Hospital, Stockport; Rajeshwar Krishnanm, Kent and Canterbury Hospital, Canterbury; Rowland Rees, University Hospital Southampton NHS Foundation Trust, Southampton; James N'dow, Aberdeen Royal Infirmary, Aberdeen; Bruce Montgomery, Frimley Park Hospital, Camberley; Michael Swinn, East Surrey Hospital, Redhill; Alastair Henderson and John Donohue, Maidstone Hospital, Maidstone; Suzie Venn, St Richard's Hospital, Chichester; Robert Mason, Torbay Hospital, Torquay; Sanjeev Madaan, Darent Valley Hospital, Kent; Mustafa Hilmy, York Hospital, York; Vivienne Kirchin, Sunderland Royal Infirmary, Sunderland; Kim Davenport, Cheltenham General Hospital, Cheltenham; John McGrath, Exeter Hospital, Exeter; Tim Porter, Yeovil District Hospital, Yeovil; Ruaraidh MacDonagh and Amerdip Birring, Musgrove Park Hospital, Taunton; Ramachandran Ravi, Basildon University Hospital; Jawad Husain, Royal Albert Edward Infirmary, Wigan; Maj Shabbir, Guy's Hospital, London; Omer Baldo, Airedale General Hospital, Keighley; Sadhanshu Chitale, Whittington Hospital, London; Mary Garthwaite, James Cook University Hospital, Middlesbrough; Shalom Srirangam, Royal Blackburn Hospital, Blackburn; Liaqat Chowoo, Bedford Hospital, Bedford; Tina Rashid, Charing Cross Hospital, London; Rob Skyrme; Jon Featherstone, Princess of Wales Hospital, Bridgend; Ammar 
Alhasso, Edinburgh; and Oleg Tatarov, Cardiff. We thank the following trusts for offering participant identification centre support: Basingstoke and Northamptonshire NHS Foundation Trust; Royal Liverpool and Broadgreen University Hospitals NHS Trust; Chelsea and Westminster NHS Foundation Trust; and Wirral University Teaching Hospital NHS Foundation Trust. We thank the following Trusts for offering PIC support: Basingstoke and Northamptonshire NHS Foundation Trust; Royal Liverpool and Broadgreen University Hospitals NHS Trust; Chelsea and Westminster NHS Foundation Trust; Wirral University Teaching Hospitals NHS Foundation Trust.

\section{Authors' contributions}

JS conduced the analysis, drafted the manuscript and approved the final draft; LV and NW drafted the manuscript and approved the final draft; BG, PW, SP critically reviewed the manuscript and approved the final draft.

\section{Funding}

Funding for this study was provided by the Health Technology Assessment programme of the National Institute for Health Research, ref: 10/57/23.

\section{Availability of data and materials}

The data used in the analysis are not publically available due to data protection, but anonymised data can be made available from corresponding author upon reasonable request.

\section{Declarations}

\section{Guidelines and regulations}

The study was conducted in line with all relevant guidelines and regulations for the conduct of a randomised controlled trial. The underlying study design was approved by the funder (UK NIHR HTA programme) in the original funding application. The detailed study protocol was approved by the Trial Steering Committee (comprising an independent clinician who acted as chairperson, two further independent clinicians, an independent statistician, a lay representative and the Chief Investigator). In addition the economic evaluation reported in this paper complied with the CHEERS checklist for the reporting of an economic evaluation.

\section{Ethics approval}

The Newcastle upon Tyne Hospitals NHS Foundation Trust Research and Development Directorate sponsored the trial (Reference 6332). Favourable ethical opinion for the trial was obtained on 16th October 2012 from the NHS Research Ethics Service Committee North East-Newcastle and North Tyneside (Reference: 12/NE/0343) and subsequent Research and Development and Caldicott approvals were granted by each participating site.

\section{Informed consent}

Study participants were consented at individual participating site following the procedure set out in the trail protocol. Trial information was provided and potential participants were given at least $48 \mathrm{~h}$ to consider participation in the study. Following review, at a mutually convenient time, those men who wanted to take part, who fulfilled the entry criteria and who understood the rationale and conduct of the trial, provided informed consent witnessed by research staff at site with delegated approval to do so. The right to refuse to participate without giving reasons was respected.

\section{Consent for publication}

Not applicable.

\section{Competing interests}

All authors declare no competing interests.

\section{Author details}

${ }^{1}$ Population Health Sciences Institute, Newcastle University, Newcastle upon Tyne, UK. ${ }^{2}$ Health Services Research Unit, University of Aberdeen, Aberdeen, UK. ${ }^{3}$ Hull York Medical School, University of Hull, Hull, UK. ${ }^{4}$ Central Manchester Hospitals NHS Foundation Trust, Manchester, UK. ${ }^{5}$ Department of Urology, St George's University of London, London, UK.

Received: 11 December 2020 Accepted: 12 April 2021

Published online: 03 May 2021

\section{References}

1. Buckley JC, Heyns C, Gilling P, Carney J. SIU/ICUD consultation on urethral strictures: dilation, internal urethrotomy, and stenting of male anterior urethral strictures. Urology. 2014;83(Suppl. 3):18-22. https://doi.org/10. 1016/j.urology.2013.08.075.

2. Pansadoro V, Emiliozzi P. Internal urethrotomy in the management of anterior urethral strictures: long-term follow up. J Urol. 1996;156:73-5.

3. Jackson MJ, Sciberras J, Mangera A, et al. Defining a patient-reported outcome measure for urethral stricture surgery. Eur Urol. 2011;60:60-8. https://doi.org/10.1016/j.eururo.2011.03.003.

4. Meeks JJ, Erickson BA, Granieri MA, Gonzalez CM. Stricture recurrence after urethroplasty: a systematic review. J Urol. 2009;182:1266-70. https:// doi.org/10.1016/j.juro.2009.06.027.

5. Chapple C, Andrich D, Atala A, et al. SIU/ICUD consultation on urethral strictures: the management of anterior urethral stricture disease using substitution urethroplasty. Urology. 2014;83(Suppl. 3):31-47. https://doi. org/10.1016/j.urology.2013.09.012.

6. Stephenson R, Carnell S, Johnson N, et al. Open urethroplasty versus endoscopic urethrotomy - clarifying the management of men with recurrent urethral stricture (the OPEN trial): study protocol for a randomized controlled trial. Trials. 2015;16:600.

7. Wessells H, Angermeier KW, Elliott S, et al. Male urethral stricture: American urological association guideline. J Urol. 2017;197:182-90.

8. Whybrow P, Pickard R, Hrisos S, Rapley T. Equipoise across the patient population: optimising recruitment to a randomised controlled trial. Trials. 2017;18(1):140.

9. Goulao B, Carnell S, Shen J, et al. Surgical treatment for recurrent bulbar urethral stricture: A randomised open label superiority trial of open urethroplasty versus endoscopic urethrotomy (The OPEN Trial). European Urology. E-pub ahead of print-4 Jul 2020

10. Briggs A, Sculpher M. An introduction to Markov modelling for economic evaluation. Pharmacoeconomics. 1998;13:397-409.

11. Consumer price index 2018 , Office of National Statistics, UK.

12. Guide to methods of technology appraisal, National Institute for Health and Care Excellence, London, 2013.

13. Briggs A, Claxton K, Sculpher M. Decision modelling for health economic evaluation. Oxford: Oxford University Press; 2006

14. Royston P. Multiple imputation of missing values. Stata J. 2004;4:227-41.

15. Brouwer W, Rutten E, Koopmanschap M. Costing in economic evaluations. In: Drummond M, McGuire A, editors. Economic evaluation in health care: merging theory and practice. Oxford: Oxford University Press; 2001.

16. Curtis LA. Unit Costs of Health and Social Care 2017. Personal Social Services Research Unit, University of Kent, Canterbury; 2017. https://doi. org/10.22024/UniKent/01.02/65559.

17. ISD Scotland. Theatre Services. 2018. www.isdscotland.org/Health-Topics/ Finance/Costs/ Detailed-Tables/Theatres.asp. Accessed 9 May 2018.

18. NHS Dictionary of Medicines and Devices. 2017.

19. Devlin NJ, Shah KK, Feng Y, Mulhern B, van Hout B. Valuing healthrelated quality of life: an EQ-5D-5L value set for England. Health Econ. 2018;2018:7-22.

20. Matthews JN, Altman DG, Campbell MJ, Royston P. Analysis of serial measurements in medical research. BMJ. 1990;300(6719):230-5.

21. Fiebig DG. Seemingly unrelated regression. In: Baltagi BH, editor. A companion to theoretical econometrics. Malden: Blackwell Publishing Ltd.; 2003. p. 101-21.

22. MacDonald S, Haddad D, Choi A, Colaco M, Terlecki R. Anterior urethroplasty has transitioned to an outpatient procedure without serious rise in complications: data from the national surgical quality improvement program. Urology. 2017;102:225-8.

23. Poelaert F, Oosterlinck W, Spinoit A-F, Lumen N. Duration of urethral catheterization after urethroplasty: how long is enough? Italian J Urol Nephrol. 2017;69:372-6.

24. Belsante MJ, Zhao LC, Hudak SJ, Lotan Y, Morey AF. Cost-effectiveness of risk stratified follow up after urethral reconstruction: a decision analysis. J Urol. 2013;190:1292-7.

25. Pickard R, Goulao B, Carnell S, Shen J, MacLennan G, Norrie J, et al. Open urethroplasty versus endoscopic urethrotomy for recurrent urethral stricture in men: the OPEN RCT. Health Technol Assess. 2020;24(61):1-110. https://doi.org/10.3310/hta24610. 
26. Shen J, Hill S, Mott D, Breckons M, Vale L, Pickard R. Conducting a time trade-off study alongside a clinical trial: a case study and recommendations. PharmacoEconomics-Open. 2019;3(1):5-20.

27. Shen J, Breckons M, Vale L, Pickard R. Using time trade-off methods to elicit short-term utilities associated with treatments for bulbar urethral stricture. PharmacoEconomics-Open. 2019;3(4):551-8.

\section{Publisher's Note}

Springer Nature remains neutral with regard to jurisdictional claims in published maps and institutional affiliations.
Ready to submit your research? Choose BMC and benefit from:

- fast, convenient online submission

- thorough peer review by experienced researchers in your field

- rapid publication on acceptance

- support for research data, including large and complex data types

- gold Open Access which fosters wider collaboration and increased citations

- maximum visibility for your research: over 100M website views per year

At BMC, research is always in progress.

Learn more biomedcentral.com/submissions 